\title{
Research and Application of wireless sensor networks in agriculture
}

\author{
Yunpeng Duan ${ }^{1, a}$, Tianjiao Guo ${ }^{2, b}$ \\ ${ }^{1}$ School of Jilin Agricultural University, Changchun 130118, China \\ ${ }^{2}$ School of Jilin Teachers Institute of engineering and technology, Changchun 130052, China \\ a9872120@qq.com, ${ }^{\mathrm{a}} \mathrm{g} \_\mathrm{t}$ j@163.com
}

Keywords: sensor, WSN, agricultural information collection, transducer, facility agriculture.

\begin{abstract}
The sensor is the main ways and means to obtain information of nature and production. In the basic study, the sensor has a more prominent position. With the rapid development of network, the development of wireless communication technology, in modern agriculture and agricultural informatization, wireless sensor network can obtain a large amount of human senses cannot directly obtain the information. Characteristics and advantages of based on wireless sensor network; this paper introduces the application of wireless sensor networks in agriculture.
\end{abstract}

\section{Introduction}

For a big country of agriculture, strengthen the application of digital technology, network technology, information technology and communication technology in agriculture, an important link of accelerating the construction of agricultural information is the information acquisition and processing. Wireless sensor network is a multi-disciplinary comprehensive technology concern, the construction of agricultural ecological monitoring system by wireless sensor grid, can be efficient real-time agricultural information collection and remote transmission, the realization of the plant diseases and insect pests, crop growth environment, soil fertility, monitoring of animal and plant physiological and ecological information, real-time and accurate measurement, thus great convenience for the work analysis of the causes and the subsequent problem solving bring, and provide a reliable basis for scientific decision making, reduce workload, improve the intelligent level of agricultural management.

\section{Definition of sensor}

The national standard GB7665-87 on the sensor's definition is: "can be provided to the feelings of measurement and according to certain rules (mathematical function law) into the device or devices available signal, usually composed of sensitive components and conversion components".

"Sensor" in the New Webster's dictionary is defined as: to receive power from a system, usually by another form of will power to devices in second systems. According to the meaning, the functions of sensor is a kind of energy into another form of energy, so many scholars also use the "transducer" to the title of "sensor".

\section{Wireless sensor network architecture}

WSN (Wireless Sensor Networks) by the deployment in the monitoring area consisting of a large number of cheap micro sensor nodes through wireless communication mode, form a multi hop and self-organized network system, its goal is the cooperative sensing, collecting and processing network coverage in the perceived object information, and then send to the observer. Wireless sensor network is typical of architecture as shown in Fig. 1. 


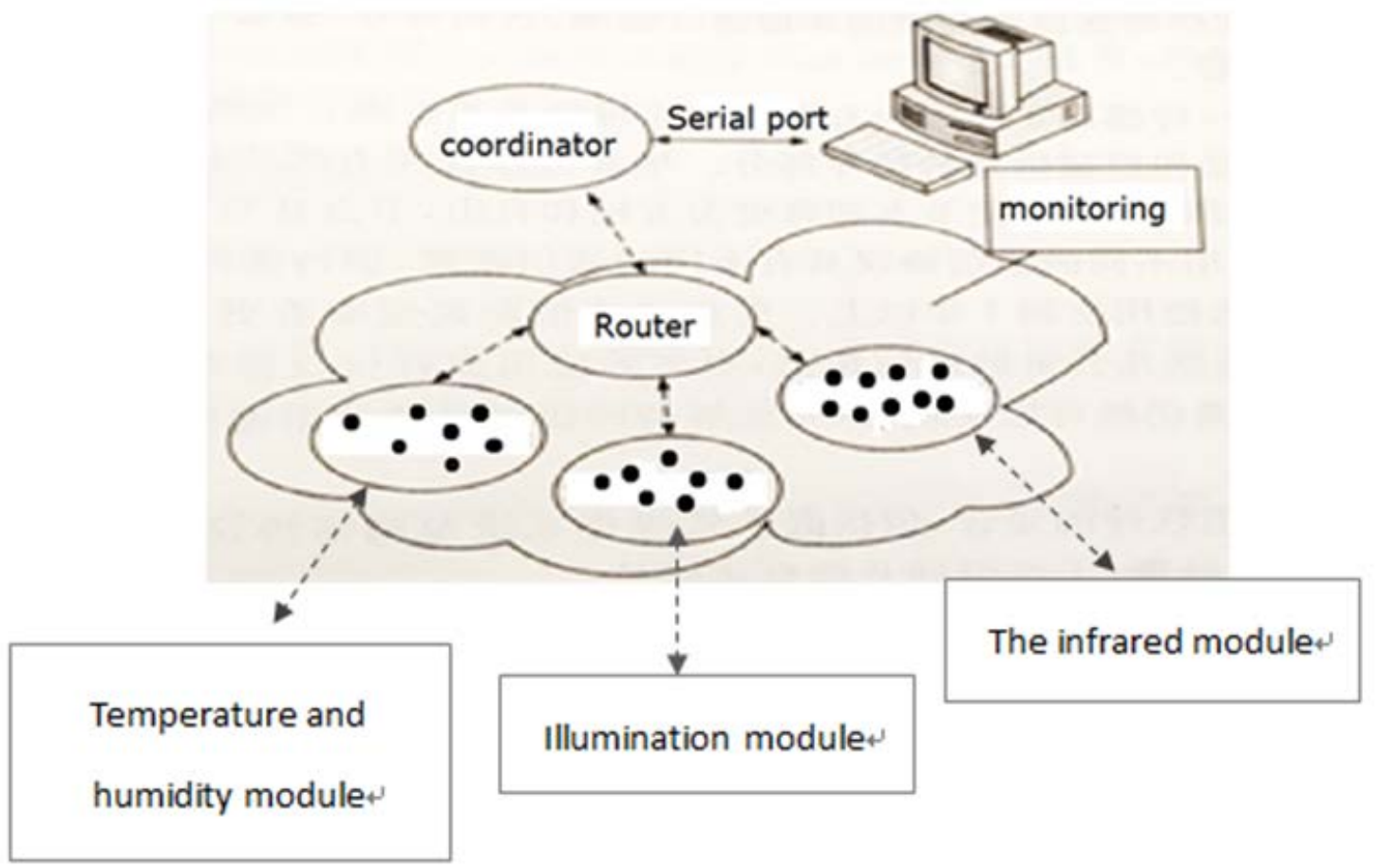

Fig.1 The wireless sensor network system structure diagram

\section{Wireless sensor network characteristics}

Wireless sensor network has the following characteristics:

(1) Node energy Co. The general use of battery powered wireless sensor nodes, limited energy, although can be supplied by solar energy technology, but as far as possible to reduce the energy consumption of the nodes and prolong the service life of the nodes is an important characteristic of WSN application.

(2) Self-organization. Nodes deployed in the monitored area, according to the automatic configuration and management of the specific application, to form a multi hop wireless network system through the topology control mechanism and network protocol.

(3) Multi hop routing. Considering reducing the energy consumption of the nodes and lines to reduce, the communication distance of nodes in the network is relatively short, generally in the range of tens to hundreds of meters, nodes can only communicate with its neighbor's direct communication. In order to let the network coverage of large area to realize the general implementation by the nodes, multi hop routing. So the wireless sensor network is a multi-hop network general.

(4) The large scale network. Dense deployment can not only reduce the requirement for a single node of the precision and reduce the cost of nodes, and a large number of the existence of redundant nodes increases the fault tolerance performance of the network, the network can be dependent on multiple nodes collaborative monitoring to improve the reliability and accuracy of monitoring.

(5) Dynamic network. Network to individual node failure, added node join, mobile node, communication environment change caused by node communication etc. automatically make the intermittent adaptation and adjustment, such as the network topology dynamically changes accordingly, ensure the effectiveness of the function of the network.

(6) A reliable network. Wireless sensor networks are often deployed in no one's field or harsh environments, sensor nodes may also suffer from the sun or wind and rain, and even irrelevant personnel or animal damage. All these require the sensor nodes is very strong, not easy to be damaged, can adapt to a variety of harsh environmental conditions

(7) Related to the application of network. According to specific application to the design of target system of wireless sensor networks, the design of efficient is the design of wireless sensor network characteristics different from traditional network. 
(8) Data centric network. The Internet is a network address as the center; general wireless sensor network is a task oriented network is reported to the user in obtaining the specified event information.

\section{Application of wireless sensor networks in agriculture}

Agriculture application is that the large number of sensor nodes constitute a monitoring network, through information collection of various sensors, to help farmers find problems in time, and accurately determine problem areas, so that agriculture will likely gradually from the human as the center, to rely on to information and software as the center of the mode of production in the isolated machinery mode of production, thus the use of a large number of all kinds of automation, intelligent, remote control production equipment.

Applied to crop growth and environmental monitoring. Put in a large number of micro sensor nodes around crop plants, is composed of sensor nodes will receive the crops growth environment factor, data transmission through the "multi hop routing will be" fused to the sink node, to realize the growth environment of region object perception on crop information collection, quantification, processing and the application of transmission.

Monitoring applied in water saving irrigation. Water saving irrigation wireless sensor networks constitute the use of a large number of soil moisture wireless sensor for real-time monitoring of the soil and control equipment sends control information to the irrigation, using the computer to the sensor information, pretreatment, and calculate the irrigation data, switch automatic control valve to achieve the electromagnetic induction, growth of demand for plant fixed-point quantitative irrigation and realize water saving, yield target.

Used in the production and storage of link monitoring of agricultural and livestock products. Through the wireless sensor network detection system arrangement of multi-tiered and wide range, for observation and Research on animals and poultry, aquaculture, life habit, rare animals, environment, physiological status and population complexity, can be found in time, the prevention and the existing problems of effective control, improve the yield and quality of livestock and poultry breeding.

Applied to agricultural acquisition. Agricultural information includes the water environment, soil environment, climate, and crop growth status of related management information and other elements, has the characteristics of large amount, multi-dimensional, dynamic, uncertain and strong temporal variability.

Should be used for facility agriculture monitoring. Application of wireless sensor network monitoring in facility agriculture is more extensive, such as in the greenhouse or shed.

In addition, also can be applied to plant diseases and insect pests of crops, poultry health status, soil PH and fertilization status, agricultural hydrology and water conservancy etc., provide powerful databases for precision agriculture.

\section{Conclusion}

Monitoring of each wireless sensor network based on its advantage of application to agricultural production aspect, can accurate, real-time data collection, wireless sensor networks with sensing, computing and communication capabilities, is an important link of modern agricultural production and management. It provides a way to obtain new information processing, information for people, for agricultural production and management in the future to automation, wireless, networking, real-time and intelligent transformation.

\section{Reference}

[1] Dun Wen Tao, Bi Qing Sheng, etc. Wireless sensor network in the application of digital agriculture [J].The world of science and technology, 2012, pp. 114, p. 123-124. 
[2] Yu Hua, Sun Yan Hong, Guo Yu Feng. Wireless sensor network application in modern agriculture [J].Anhui agricultural science, 2010, p. 2172-2174.

[3] Qiao Xiao Jun, Zhang Xin, Wang Cheng, etc. Wireless sensor network application in agriculture [J].Journal of agricultural engineering, 2005, p. 232- 234.

[4] Peng Xiao Dong, Zhang Tie min, etc. Application of wireless sensor networks in agriculture [J].Journal of agricultural engineering, 2011, p. 245-248.

[5] Li Hai Jian, Wang Min, Wang Huai De, Zhang Yi Wireless sensor network application in farmland information collection [J].Agricultural mechanization research, 2008, p. 187-189.

[6] Zheng Shao Xing. Wireless sensor networks in agriculture information in the application of [J]. Digital technology and application, 2012, p. 85-86. 